\title{
Disease-Specific Markers for the Mucopolysaccharidoses
}

\author{
MARIA FULLER, TINA ROZAKLIS, STEVEN L. RAMSAY, JOHN J. HOPWOOD, AND \\ PETER J. MEIKLE
}

\begin{abstract}
Lysosomal Diseases Research Unit [M.F., T.R., S.L.R., J.J.H., P.J.M.], Department of Genetic Medicine, Women's and Children's Hospital, North Adelaide, 5006, South Australia, Australia; and Department of Paediatrics [M.F., J.J.H., P.J.M.], University of Adelaide, Adelaide, 5005, South Australia, Australia
\end{abstract}

\begin{abstract}
ABST
Unprecedented demands are now placed on clinicians for
early diagnosis as we enter into an era of advancing treatment
opportunities for the mucopolysaccharidoses (MPS). Biochemi-
cal monitoring of any therapeutic avenue will also be prerequi-
site. To this end, we aimed to identify a range of urinary
oligosaccharides that could be used to identify and characterize
patients with MPS. We analyzed 94 urine samples from 68
patients with MPS and 26 control individuals for oligosaccha-
rides derived from glycosaminoglycans using electrospray ion-
ization-tandem mass spectrometry. The oligosaccharide profile
for each patient group was compared with that of the control
group. The Mann-Whitney $U$ test was used to measure the
difference between each patient group and the controls for each
analyte. Urine samples from patients before and at successive
times after bone marrow transplantation were also evaluated. A
number of oligosaccharides were identified in the urine of each
MPS subtype, and for each of these, specific oligosaccharide
profiles were formulated. These profiles enabled the identifica-
tion of all 68 patients and their subtypes with the exception of
\end{abstract}
\section{ABSTRACT}

MPS IIIB and IIIC. Selected oligosaccharides were used to assess three individuals after a bone marrow transplant, and, in each case, a substantial reduction in the level of diagnostic oligosaccharides, posttransplantation, was observed. The identification and measurement of glycosaminoglycan-derived oligosaccharides in urine provides a sensitive and specific screen for the early identification of individuals with MPS. The resulting oligosaccharide profiles not only characterize subtype but also provide a disease-specific fingerprint for the biochemical monitoring of current and proposed therapies. (Pediatr Res 56: 733738, 2004)

\[ \text { Abbreviations } \]
ESI-MS/MS, electrospray ionization-tandem mass
spectrometry
GAG, glycosaminoglycans
HN, hexosamine
MPS, mucopolysaccharidoses
UA, uronic acid

The mucopolysaccharidoses (MPS) are a group of inherited lysosomal storage disorders characterized by a deficiency in one of the lysosomal enzymes required to degrade glycosaminoglycans (GAG). There are 11 known enzyme deficiencies that give rise to seven distinct types of MPS, with a combined incidence of $\sim 1$ in 16,000 (1). In all MPS subtypes, partially degraded GAG accumulate in the lysosomes of affected cells and/or are excreted in the urine. The lysosomal storage of GAG leads to the chronic and progressive deterioration of cells, tissues, and organs (2). The MPS share many clinical manifestations, including organomegaly, abnormal facial features, and

Received November 26, 2003; accepted April 20, 2004

Correspondence: Maria Fuller, Lysosomal Diseases Research Unit, Department of Genetic Medicine, Women's and Children's Hospital, 72 King William Road, North Adelaide, 5006, South Australia, Australia; e-mail: maria.fuller@adelaide.edu.au

This work was supported by the National Health and Medical Research Council of Australia, The Wellcome Trust (UK) grant reference number 060104Z/00/Z and TLH Research.

DOI: 10.1203/01.PDR.0000141987.69757.DD dysostosis multiplex. Impaired hearing, vision, and joint mobility, as well as abnormal airway and cardiovascular function, are common, although there is wide clinical heterogeneity within each enzyme deficiency. Profound mental retardation is characteristic of the severe forms of MPS I, II, and VII and all subtypes of MPS III. Similar and severe skeletal and joint abnormalities are present in MPS I, II, VI, and VII, whereas MPS IV develops different skeletal pathology.

The clinical management for MPS is changing, as new treatment options, such as enzyme replacement therapy, that will complement and replace bone marrow transplantation undergo trials. Enzyme replacement therapy is currently available for MPS I $(3,4)$, and clinical trials are in progress for MPS II and VI. Bone marrow transplantation has been used successfully for severe MPS I patients for $>20 \mathrm{y}$. However, for therapy to achieve a good long-term outcome, particularly for MPS involving central nervous system (CNS) and bone pathology, treatment before the onset of irreversible pathology will be an important benchmark. In addition, prediction of 
disease severity will play a pivotal role in deciding on therapeutic alternatives. Enzyme replacement therapy is likely to treat only somatic pathology, whereas bone marrow transplantation, if performed early enough, will have an impact on CNS pathology (5). Similarly, the efficacy gained from enzyme replacement therapy in MPS VI cats has been shown to benefit by commencing therapy at birth (6). Earlier identification and provision of a prognosis for MPS patients, paralleled with the availability of multiple therapy options, should enable the commencement of therapy before the onset of serious and irreversible clinical symptoms. When therapy is commenced before the onset of clinical symptoms and in the absence of comprehensive natural history studies, biochemical markers of disease activity will be mandatory to evaluate the efficacy of therapy and will be useful for the determination of dosage. To address the need for early diagnosis, prognosis, and an ability to monitor therapy for MPS, we aimed to characterize a biochemical picture of disease activity. Using electrospray ionization-tandem mass spectrometry (ESI-MS/MS), we identified a range of sulfated oligosaccharides, derived from the partial degradation of GAG, in urine from MPS patients. For the most part, these oligosaccharides have a nonreducing terminus that consists of the specific substrate for the deficient enzyme in that particular MPS disorder. It is thought that they arise from endolytic cleavage of larger GAG followed by exolytic trimming to the resistant structures observed.

\section{METHODS}

Derivatization of urine samples. Urine samples were submitted to the Women's and Children's Hospital for diagnosis, used with consent, and the hospital ethics committee approved this study. Urine samples $(0.5-\mu \mathrm{mol}$ creatinine equivalents) were lyophilized and then resuspended in $100 \mu \mathrm{L}$ of $250 \mathrm{mM}$ of 1-phenyl-3-methyl-5-pyrazolone, $400 \mathrm{mM}$ of $\mathrm{NH}_{4} \mathrm{OH}$ containing $1 \mathrm{nmol}$ of deuterated $\mathrm{N}$-acetylglucosamine-6-sulphate $\left(\right.$ GlcNAc6S- $d_{3}$ ) as described previously (7), and $2 \mathrm{nmol}$ of 4-deoxy-L-threo-hex-4-enopyranosyluronic $(1 \rightarrow 3)$ N-acetylgalactosamine-4-sulphate $(\Delta \mathrm{UA}-\mathrm{GalNAc} 4 \mathrm{~S})$ as internal standards. The derivatization was performed at $70^{\circ} \mathrm{C}$ for $90 \mathrm{~min}$ and then acidified with a 2 -fold molar excess of $\mathrm{HCO}_{2} \mathrm{H}$. Samples were made up to $500 \mu \mathrm{L}$ with $\mathrm{H}_{2} \mathrm{O}$ and then extracted with an equal volume of $\mathrm{CHCl}_{3}$ to remove excess 1-phenyl-3methyl-5-pyrazolone and centrifuged at 13,000 $\times g$ for $5 \mathrm{~min}$. Solid-phase extraction cartridges ( $25 \mathrm{mg}$ of $\mathrm{C} 18)$ were primed with $1 \mathrm{~mL}$ each of $\mathrm{CH}_{3} \mathrm{OH}$ and then $\mathrm{H}_{2} \mathrm{O}$, after which the sample was applied and allowed to enter the solid phase. Samples were desalted with three consecutive $1 \mathrm{~mL} \mathrm{H}_{2} \mathrm{O}$ washes and dried on a Supelco Visiprep24 vacuum manifold, and any remaining 1-phenyl-3-methyl-5-pyrazolone was removed with two $\mathrm{CH}_{3} \mathrm{Cl}$ washes. The columns were again dried, and derivatized oligosaccharides were eluted in an aqueous solution of $50 \%$ (vol $/ \mathrm{vol}$ ) $\mathrm{CH}_{3} \mathrm{CN} / 0.025 \%$ (vol $/ \mathrm{vol}$ ) $\mathrm{HCO}_{2} \mathrm{H}$.

Mass spectrometry. Mass spectrometry was performed on a PE Sciex API 3000 triple-quadruple mass spectrometer with a turbo-ionspray source $\left(200^{\circ} \mathrm{C}\right)$ and Analyst 1.1 data system. Samples $(20 \mu \mathrm{L})$ were injected into the electrospray source with a Gilson 233 autosampler using a carrying solvent of $50 \%$
$\mathrm{CH}_{3} \mathrm{CN} / 0.025 \% \mathrm{HCO}_{2} \mathrm{H}$ in $\mathrm{H}_{2} \mathrm{O}$ at a flow rate of $80 \mu \mathrm{L} / \mathrm{min}$. $\mathrm{N}_{2}$ was used as the collision gas at a pressure of $2 \times 10^{-5}$ Torr and for the nebulizing process. Quantification of 1-phenyl-3methyl-5-pyrazolone-derivatized oligosaccharides was performed using multiple-reaction monitoring in negative ion mode. Each ion pair was monitored for $100 \mathrm{~ms}$ with a resolution of $1 \mathrm{amu}$ at half-peak height and averaged from continuous scans over the injection period. Relative oligosaccharide levels were calculated by relating the peak heights of the 1-phenyl3-methyl-5-pyrazolone-oligosaccharides to the peak height of the internal standard. The GlcNAc6S- $d_{3}$ was used as the internal standard for the monosaccharides; di- and larger oligosaccharides were related to $\triangle \mathrm{UA}-\mathrm{GalNAc} 4 \mathrm{~S}$.

Statistical analysis. The Mann-Whitney $U$ test was used to measure the difference between each patient group and the control for each analyte.

\section{RESULTS}

A total of 26 oligosaccharides was identified in the urine from a number of MPS and control individuals. They ranged in size from mono- to hexasaccharides consisting of alternating uronic acid (UA) and hexosamine (HN) residues with varying degrees of sulfation and $\mathrm{N}$-acetylation. Proposed structures for these oligosaccharides were suggested on the basis of massto-charge ratios and fragmentation patterns from ESI-MS/MS. Of these 26 oligosaccharides, 10 were shown to be required for the diagnosis of an MPS (Table 1). For every MPS subtype, at least one analyte-but in the majority of instances, a number of analytes-is significantly elevated or, in one case, decreased relative to the control population. This enabled complete discrimination of each MPS subtype from unaffected individuals.

Figure $1 A$ shows the relative levels of the 10 oligosaccharides and their variation in the 26 control samples analyzed. Figure $1 B$ shows a characteristic profile of these 10 oligosaccharides in each of the MPS subtypes. The 15 MPS I patients all could be distinguished by the high levels of a monosulfated di- and trisaccharide [UA-HNAc(S) and UA-HNAc-UA(S)]. These di- and trisaccharides were also elevated in the 13 MPS II patients, but the increase of a hexasaccharide (unknown structure), at proportionately higher levels than the di- and trisaccharide in these patients, enabled discrimination of MPS II from MPS I. MPS IIIA and IIIB patients were easily identified by increased levels of a heparan sulphate-derived disaccharide (HNS-UA) and tetrasaccharide [HNAc-UAHNAc-UA(S)], respectively. There were four MPS IIIC patients included in this study, but only two of these could be discriminated from the control population using the tetrasaccharide [(HNAc-UA)2(S)]. In the other two patients, all analytes were in the normal range; however, the ratio of the hexasaccharide (unknown structure) to the trisaccharide [UA$\mathrm{HN}-\mathrm{UA}(\mathrm{S}) 2]$ delineated all four patients from the control population. Nevertheless, we were unable to differentiate the MPS IIIB from the IIIC subtypes. MPS IIID, IVA, and VI all have previously been identified using a sulfated $\mathrm{N}$-acetylhexosamine (HNAcS) $(7,8)$. The MPS VI patients could be distinguished from IVA and IIID patients by an elevated ratio of the monosulfated to disulfated monosaccharide (HNAcS2). 
Table 1. Mann Whitney U values for oligosaccharide analytes in MPS subgroups compared with control subjects $(\mathrm{n}=26)$

\begin{tabular}{|c|c|c|c|c|c|c|c|c|}
\hline & MPS I & MPS II & MPS IIIA & MPS IIIB & MPS IIIC & MPS IIID & MPS IVA & MPS VI \\
\hline$n$ & 15 & 12 & 12 & 7 & 4 & 2 & 6 & 10 \\
\hline 1 HNAcS $(630.4 / 256.1)$ & $73 *$ & 99 & $67^{*}$ & 53 & 27 & $0 \dagger$ & $0^{*}$ & $0^{*}$ \\
\hline 2 HNAcS2 (710/256) & $85^{*}$ & 121 & $60^{*}$ & 58 & 22 & 14 & $30 \dagger$ & $7 *$ \\
\hline 4 UA-HNAcS $(806 / 295)$ & $0^{*}$ & $0^{*}$ & 120.5 & 80 & 42 & $2 \dagger$ & 67 & 115 \\
\hline 5 HNAcS-UA $(806 / 331)$ & 194 & 151 & 154 & 76 & 38 & $0 \dagger$ & $1 *$ & $4 *$ \\
\hline 6 UA-HNAc-UA(S) $(490.5 / 476)$ & $0^{*}$ & $8^{*}$ & 140 & 51 & 36 & 23 & 67.5 & 128 \\
\hline 9 (HNAcS-UA)2 (632.3/298) & $5^{*}$ & 123 & $3^{*}$ & $5^{*}$ & 28 & $0 \dagger$ & $10.5^{*}$ & $0 *$ \\
\hline 10 hexasaccharide $(764.8 / 256.1)$ & $0 *$ & $0 *$ & $13 *$ & $1 *$ & $3^{*}$ & $0 \dagger$ & $2 *$ & $8 *$ \\
\hline
\end{tabular}

The relative levels of each of the 10 oligosaccharides were determined by ESI-MS/MS using the multiple-reaction monitoring pairs listed in parentheses. The Mann Whitney $U$ values are reported for the 10 oligosaccharides for each MPS subtype compared with the unaffected control subjects.

$* p<0.01$.

$\dagger p<0.05$.

Only two MPS IIID patients were included in this study, and they could be distinguished from the IVA patients by an elevation in the HNS-UA with a Mann-Whitney $U$ value of 0 $(p<0.05)$.

Figure 2 shows the relative levels of selected markers in MPS I, IVA, and VI patients who had been treated with bone marrow transplantation. All patients showed a substantial reduction in the level of diagnostic oligosaccharides with time after transplantation. The urine samples from the MPS I patient who received a bone marrow transplant were also assayed for total urinary GAG (9). A concomitant decrease of the oligosaccharides measured in MPS I was observed with total urinary GAG levels.

\section{DISCUSSION}

Cell death and/or exocytosis results in the appearance of high molecular weight GAG and GAG-derived oligosaccharides in the urine of MPS patients at increased levels over that seen in unaffected individuals. These oligosaccharides are probably a consequence of endolytic cleavage of the high molecular weight GAG chains. The nature of the oligosaccharides that are elevated in each MPS disorder is primarily a reflection of the block in catabolism as a result of the enzyme deficiency. The sulfated disaccharide $[\mathrm{UA}-\mathrm{HNAc}(\mathrm{S})]$ and trisaccharide [UA-HNAc-UA(S)] seen in MPS I contain $\alpha$-Liduronic acid at the nonreducing end as determined by digestion with $\alpha$-L-iduronidase (data not shown). The presence of these oligosaccharides is a direct consequence of the deficiency in $\alpha$-L-iduronidase. In MPS II, oligosaccharides with the same composition are elevated, but they are likely to be structural isomers with the sulphate on the terminal nonreducing $\alpha$-Liduronic acid. The marker used to identify MPS IIIA patients was an HNS-UA, whereas the marker specific for MPS IIIB was a monosulfated tetrasaccharide [(HNAc-UA)2(S)], both showing a nonreducing end specific for the deficient enzyme. We were unable to differentiate the MPS IIIB from the IIIC patients, although it is noteworthy that the incidence of MPS IIIB is seven times higher than that for IIIC (1). Further work will be required to identify oligosaccharides specific for these two subtypes. MPS IIID, IVA, and VI store GAG with nonreducing end, $\beta$-linked, sulfated $\mathrm{N}$-acetylhexosamine (HNAcS).
The elevated level of HNAcS in these patients is due to an alternate degradation pathway in which $\beta$-N-acetylhexosaminidase cleaves the nonreducing end sulfated $\mathrm{N}$ acetylhexosamine (8). The disulfated monosaccharide (HNAcS2) was also elevated in MPS VI patients, as the $\mathrm{N}$-acetylgalactosamine-4-sulphatase action precedes $\mathrm{N}$-acetylgalactosamine-6-sulphatase action in the hydrolysis of the HNAcS2 $(7,8)$. No urine samples were available from MPS VII patients, but an unsulfated trisaccharide (UA-HNAc-UA) has been shown to be elevated in amniotic fluids from affected fetuses and as such should provide a diagnostic marker for MPS VII (unpublished observations). In addition, some oligosaccharides may result from secondary storage, in which lysosomal function is impaired to such a degree that oligosaccharides with nonreducing ends not reflective of the enzyme

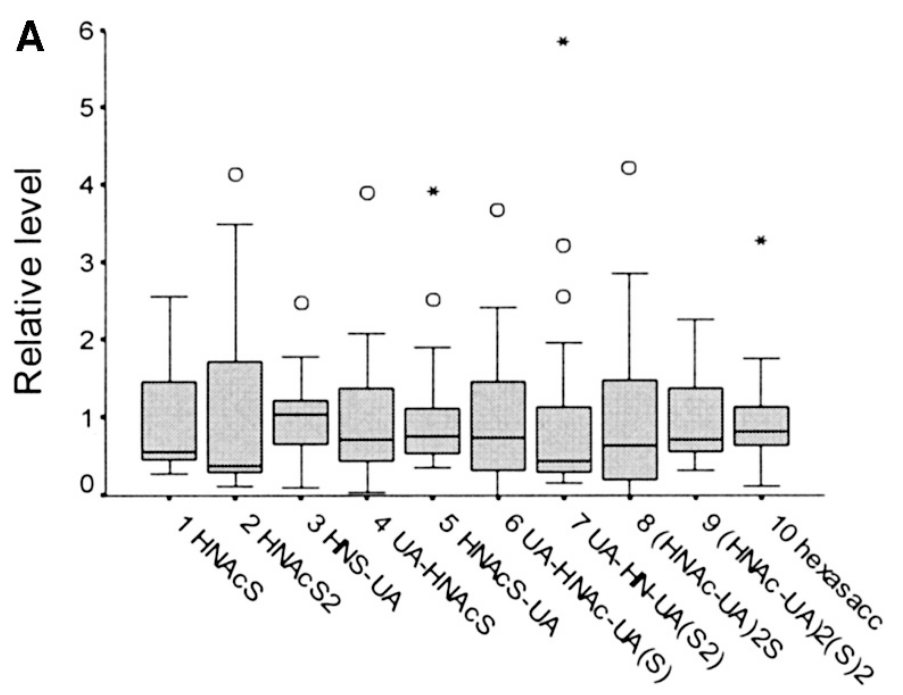

Figure 1. Relative levels of GAG-derived oligosaccharides in control and MPS individuals. Urine samples from 26 control samples $(A)$ and 68 MPS individuals representing eight subtypes $(B)$ were analyzed for $10 \mathrm{GAG}$-derived oligosaccharides. The results were normalized to the control population in which the average value for each analyte was assigned a value of 1 . The box plot shows the median level of each analyte in the control group and each MPS subtype (center bar), the 25th and 75th centiles (boxes), and the upper and lower limits (upper and lower bars). The circles and asterisks represent outliers and extreme outliers, respectively. 

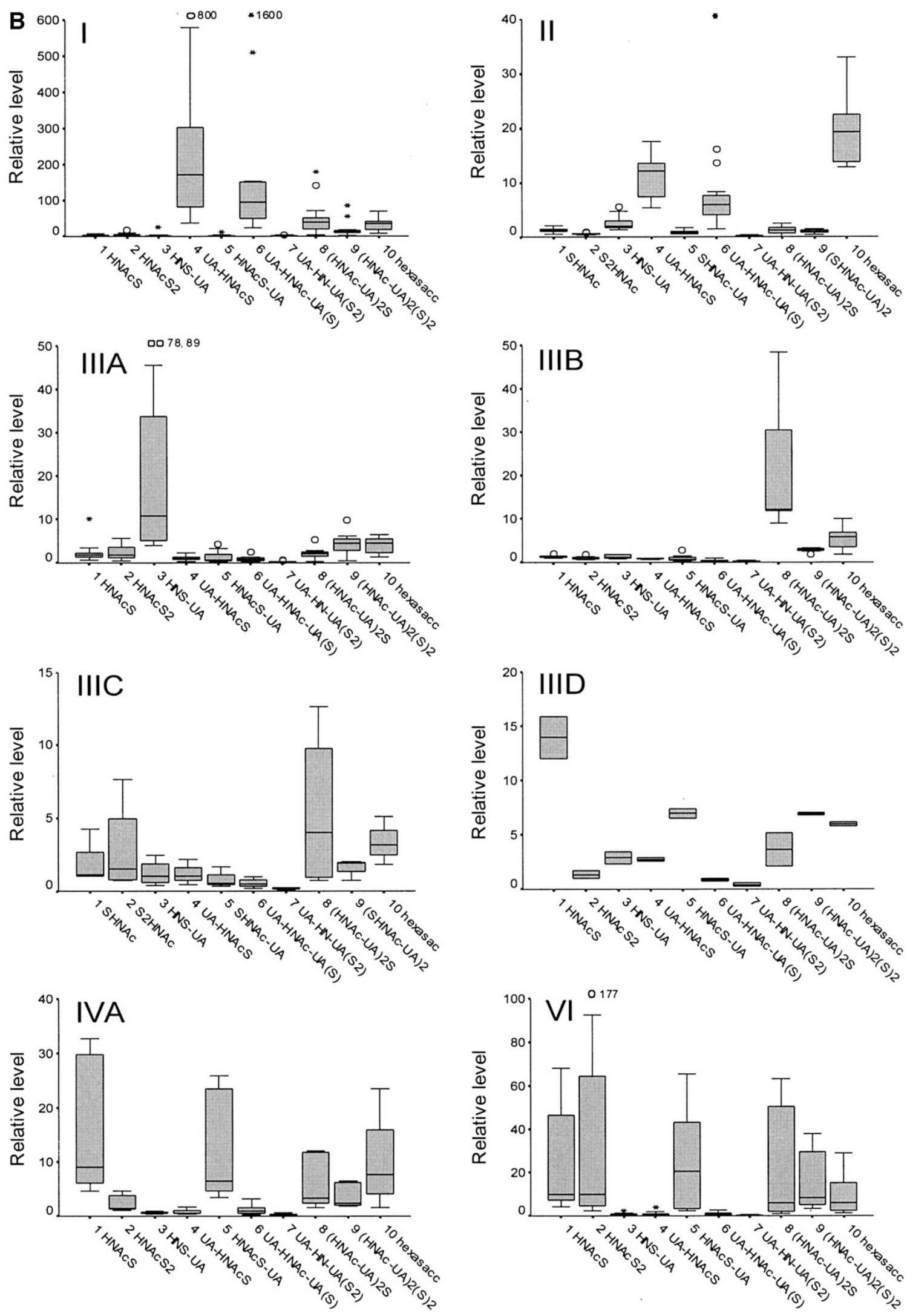

Figure 1. Continued 

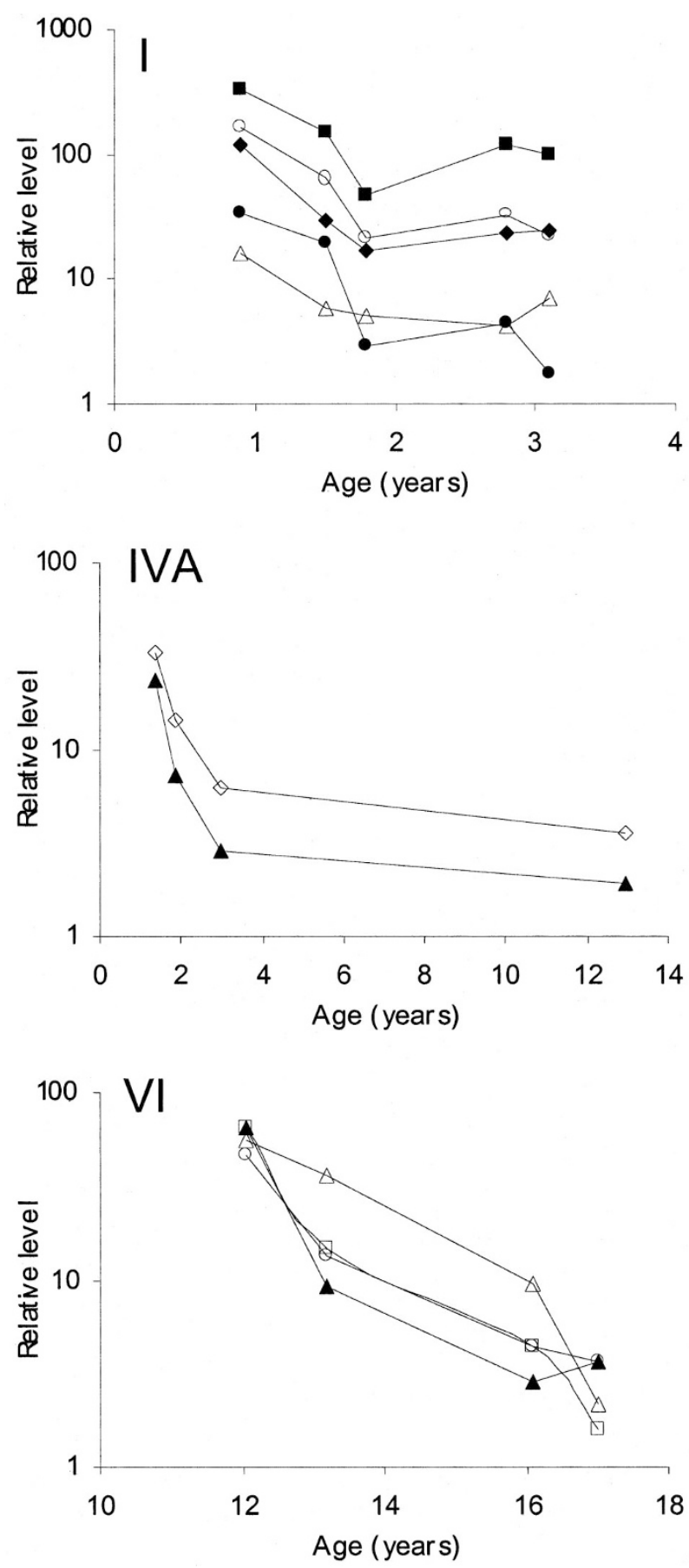

Figure 2. Relative levels of GAG-derived oligosaccharides in urine from MPS-affected individuals before and after bone marrow transplant. Urine samples from MPS I, MPS IVA, and MPS VI patients were collected before transplant and at successive times after transplantation. Each sample was analyzed for GAG-derived oligosaccharides as described, and the results were normalized to the control population, in which each analyte was assigned a value of 1 . The MPS I, IVA, and VI patients received bone marrow transplants at 1.2, 1.9, and $12.1 \mathrm{y}$ of age, respectively. Oligosaccharides monitored in each patient were total urinary GAG $(\diamond), \operatorname{HNAcS}(\diamond), \operatorname{HNAcS} 2(\square)$, UA-HNAcS

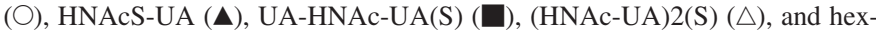
asaccharide $(\mathbf{O})$.

deficiency accumulate. Nonetheless, they seem to be a minority.

In the absence of a family history, diagnosis of MPS can be a long and drawn-out process. Presymptomatic diagnosis for
MPS has been proposed through newborn screening programs but has been thwarted by a lack of appropriate technology (10). The use of ESI-MS/MS has enabled the measurement of GAG-derived oligosaccharides from small amounts $(\sim 100 \mu \mathrm{L})$ of urine to definitively diagnose each MPS type. This type of urine screen is amenable to high throughput and reduces the need for expensive, time-consuming enzymology. Confirmation of the subtype would be required by enzymology but would involve only one enzyme assay. The only exception would be in the instance of MPS IIIB and IIIC, which cannot be delineated and therefore would require two enzyme assays. Extension of this approach into dried blood spots may have application in newborn screening programs for these disorders as either a primary screen or a confirmation test subsequent to a screen.

The potential applicability of these oligosaccharides to monitor effectiveness of therapy regimens was demonstrated by analyzing urine samples from three patients who had been treated by bone marrow transplantation. Both the MPS IVA and VI patients showed a concomitant decrease in HNAcS with time after transplant, with a similar reduction in HNAcS2 in the MPS VI patient. A number of other primary and possibly secondarily stored oligosaccharides were also shown to decrease after bone marrow transplantation in these patients (Fig. 2 ), with all analytes approaching normal (a level of 1) within 2-3 y after transplantation. The MPS I patient, with the genotype W402X/Q70X, is predicted to develop a severe phenotype and showed reductions in the primary oligosaccharides [UAHNAcS and UA-HNAc-UA(S)] over the first 2 y after transplant. Total GAG levels were also measured on the MPS I patient and shown to follow the same trend as the individual oligosaccharides (Fig. 2). Progression of the pathology in this MPS I patient has not continued as predicted from the patient's genotype. These are preliminary results, and follow-up of these and additional patients will be required to fully validate these oligosaccharide markers for the monitoring of therapy for MPS.

The continued development of therapies for MPS raises additional issues. A major hurdle facing clinical trials for enzyme replacement therapy resides in the inability to adequately monitor their efficacy. There is considerable heterogeneity within each MPS subtype even between patients with the same genotype (11), making not only prediction of disease progression arduous but also comparative reference points for clinical trials almost impossible. We observed a correlation in the amount of these oligosaccharides with genotype in a few instances. The levels of most of the oligosaccharides from MPS I patients with genotypes (W402X homozygous and W402X/Q70X) that are known to lead to severe clinical phenotypes were higher than the levels of the same oligosaccharides detected in urine from MPS I patients with mutations (e.g. R89Q) that are known to lead to milder phenotypes (12). Furthermore, a patient whose MPS IIIA was diagnosed in the fourth decade of life, with very mild symptoms (13), was shown to have very low levels of the heparan sulphate-derived disaccharide. Notwithstanding these initial observations, creatinine is derived from muscle metabolism and levels increase with age; therefore, for phenotype predictions using this ap- 
proach, affected individuals should be compared with agematched control subjects. We believe that with continued evaluation, GAG-derived oligosaccharides and the metabolic profiles generated by their determination will be instrumental in the clinical management of this diverse and complex group of disorders.

\section{REFERENCES}

1. Meikle PJ, Hopwood JJ, Clague AE, Carey WF 1999 Prevalence of lysosomal storage disorders. JAMA 281:249-254

2. Neufeld EF, Muenzer J 2001 The mucopolysaccharidoses. In: Scriver CR, Beaudet AL, Sly WS, Valle D (eds) The Metabolic and Molecular Bases of Inherited Disease. McGraw-Hill, New York, pp 3421-3452

3. Kakkis ED, Muenzer J, Tiller GE, Waber L, Belmont J, Passage M, Izykowski B Phillips J, Doroshow R, Walot I, Hoft R, Neufeld EF 2001 Enzyme-replacemen therapy in mucopolysaccharidosis I. N Engl J Med 344:182-188

4. 2002 Laronidase. Biodrugs 16:316-318

5. Whitley CB, Belani KG, Chang PN, Summers CG, Blazar BR, Tsai MY, Latchaw RE, Ramsay NK, Kersey JH 1993 Long-term outcome of Hurler syndrome following bone marrow transplantation. Am J Med Genet 46:209-218
6. Crawley AC, Niedzielski KH, Isaac EL, Davey RC, Byers S, Hopwood JJ 1997 Enzyme replacement therapy from birth in a feline model of mucopolysaccharidosis type VI. J Clin Invest 99:651-662

7. Ramsay SL, Meikle PJ, Hopwood JJ 2003 Determination of monosaccharides and disaccharides in mucopolysaccharidoses patients by electrospray ionisation mass spectrometry. Mol Genet Metab 78:193-204

8. Hopwood JJ, Elliott H 1985 Urinary excretion of sulphated N-acetylhexosamines in patients with various mucopolysaccharidoses. Biochem J 229:579-586

9. de Jong JGN, Wevers RA 1992 A modified DMB-Tris assay. Clin Chem 38:803-807

10. Meikle PJ, Brooks DA, Ravenscroft EM, Yan M, Williams RE, Jaunzems AE, Chataway TK, Karageorgos LE, Davey RC, Boulter CD, Carlsson SR, Hopwood JJ 1997 Diagnosis of lysosomal storage disorders: evaluation of lysosome-associated membrane protein LAMP-1 as a diagnostic marker. Clin Chem 43:1325-1335

11. Scott HS, Nelson PV, Litjens T, Hopwood JJ, Morris CP 1993 Multiple polymorphisms within the $\alpha$-L-iduronidase gene (IDUA): implications for a role in the modification of MPS-I disease phenotype. Hum Mol Genet 2:1471-1473

12. Scott HS, Bunge S, Gal A, Clarke LA, Morris CP, Hopwood JJ 1995 Molecular genetics of mucopolysaccharidosis type I: diagnostic, clinical and biological implications. Hum Mutat 6:288-302

13. Van Hove JL, Wevers RA, Van Cleemput J, Moerman P, Sciot R, Matthijs G, Schollen E, de Jong JG, Carey WF, Muller V, Nicholls C, Perkins K, Hopwood JJ 2003 Late-onset visceral presentation with cardiomyopathy and without neurological symptoms of adult Sanfilippo A syndrome. Am J Med Genet 118A:382-387 